a mismatch between a current sound and the memory formed of a repetitious sequence. MMN can be conceptualized as occurring when a sound violates the context established by preceding events. Individuals with schizophrenia are known to show impairments in the ability to use context when processing the relevance of an event. In this study, we examined whether this impairment in using context would result in insensitivity to the effects of temporal context on MMN. Specifically, we explore how temporal context affects the MMN produced by healthy controls and individuals with schizophrenia.

Method: Eighteen patients and age- and sex-matched controls were presented with two sound sequences over headphones, with attention directed toward a silent movie with subtitles. In the Fixed stimulus onset asynchrony (SOA) sequence, MMN was measured to a 100-ms duration deviant among 50-ms standard tones at a regular 500-ms SOA. The same tones were used in the jittered SOA sequence, with irregular SOAs ranging from 250 to $750 \mathrm{~ms}$.

Results: In controls, the MMN elicited by duration deviants with jittered SOA was significantly reduced compared with the same deviant occurring in a sequence with fixed SOA. However, changing temporal context/regularity had no significant impact on the MMN produced by the schizophrenia group.

Conclusions: The results indicate that individuals with schizophrenia have difficulty encoding and forming a model of the temporal context in which a sound occurs.

\section{Research priorities in suicide prevention}

\section{J Robinson'1, E Schindeler², M Dudley², D De Leo³, T Jorm', S Harrigan', S Niner ${ }^{4}$, K Krysinska3 ${ }^{3}$ J Pirkis ${ }^{4}$}

'ORYGEN Research Centre; ${ }^{2}$ Suicide Prevention Australia; ${ }^{3}$ Australian Institute for Suicide Research and Prevention; and PProgram Evaluation Unit,

School of Population Health, The University of Melbourne, Melbourne, Australia

Background: Consistent with the evidence-based approach of the National Suicide Prevention Strategy and the goals of the LIFE Framework, the Commonwealth Department of Health and Ageing has funded a project designed to identify future areas of research to be addressed in suicide prevention.

Aims: The project aims to identify current priorities, as evidenced by research that has been funded and published over the course of the strategy (19992006), and to identify future priorities, by considering the gaps in existing research and by consulting with stakeholders.
Methods: There are four components to the project: 1) a systematic review of the published literature, 2) a review of suicide prevention research projects funded by key granting bodies, 3 ) a series of stakeholder focus groups and 4) a questionnaire for representatives from key stakeholder groups.

Results: Initial findings indicate that the majority of published literature is epidemiological, focusing on rates of suicide. There is little intervention research and little program or service evaluation. In contrast, the majority of funded projects are intervention studies. With regard to stakeholder priorities, several recurring themes emerged including the need for evaluating the efficacy of interventions, necessity of balancing individual risk factors with societal influences on suicide and the need for consideration of protective factors. The results from each component of the project will be presented in more detail.

Conclusions: The implications of these findings for future suicide prevention research will be discussed and recommendations for a national research agenda will be made.

\section{Adaptation of a resilience program in NSW: the rural RAP}

\section{T Robinson \\ Centre for Rural and Remote Mental Health, Orange, Australia}

Aim: To document the process of adapting an emotional resilience program for indigenous students.

Background: There are a number of programs in schools to enhance the emotional resilience of young people, but few studies have adapted programs for use with indigenous youth. During 2005, the Resourceful Adolescent Program (RAP) was adapted in collaboration with Aboriginal workers and community members from Bourke, Dubbo and Wellington prior to its implementation in four local high schools. This presentation will provide an overview of the adaptation process.

Methods: A steering committee comprising cultural consultants, representatives from the Department of Education and health workers oversaw the adaptation of RAP. An overarching participatory action framework was used to facilitate inductive knowledge about the adaptation of RAP.

Results: A total of 26 Aboriginal education consultants, assistants, tutors and community members participated in the adaptation and implementation of RAP. In terms of content, the overarching metaphor for well-being was adapted for rural youth and self-esteem activities were broadened to include cultural identity. In addition, process issues about small group facilitation with Aboriginal students were 